\title{
Impact of dexamethasone, etoposide, ifosfamide and carboplatin as concurrent chemoradiotherapy agents for nasal natural killer/T-cell lymphoma
}

\author{
YOSHIOMI HATAYAMA, MASAHIKO AOKI, HIDEO KAWAGUCHI, YUICHIRO NARITA, \\ KATSUMI HIROSE, MARIKO SATO and YOSHIHIRO TAKAI
}

Department of Radiology, Hirosaki University School of Medicine, Hirosaki, Aomori 036-8562, Japan

Received December 20, 2012; Accepted April 23, 2013

DOI: $10.3892 /$ mco.2013.123

\begin{abstract}
The nasal type of extranodal natural killer $(\mathrm{NK}) / \mathrm{T}$-cell lymphoma (NKTCL) is a rare aggressive lymphoma with poor prognosis. The reported 5-year overall survival for patients with localized nasal NKTCL treated with cyclophosphamide, hydroxydaunorubicin, oncovin and prednisone (CHOP) is $<50 \%$. Dexamethasone, etoposide, ifosfamide and carboplatin (DeVIC) chemotherapy was designed as a salvage chemotherapeutic regimen for aggressive lymphoma, comprising multidrug resistance (MDR) non-related agents and etoposide, which is considered to be effective against nasal NKTCL. An experimental chemoradiotherapy (CRT) is currently being designed using DeVIC as the concurrent chemotherapeutic regimen for nasal NKTCL. The aim of this study was to examine the initial outcome of this treatment and evaluate its effectiveness and feasibility. Six patients (age range, 29-82 years; median age, 68 years) were treated with CRT using DeVIC between April, 2004 and February, 2010. The median follow-up was 56 months (range, 11-80 months). All patients were administered 3-6 cycles of full-dose DeVIC regimen. The chemotherapy was administered concurrently with radiotherapy (RT) and was repeated every 3 weeks. RT was performed using 4-MV X-ray and the prescription dose was 46-50 Gy/23-25 fx (median, 50 Gy). After treatment, all patients were followed up at our hospital. A complete remission was achieved in 5 patients $(83 \%)$ at 1 month after treatment. The 5-year overall survival and disease-free survival rates were $100 \%$. No severe adverse effect (grade $\geq 3$ ) was reported. In conclusion, the initial results of the experimental CRT with DeVIC for this type of aggressive lymphoma were very encouraging. Further investigation is required on concurrent
\end{abstract}

Correspondence to: Dr Yoshiomi Hatayama, Department of Radiology, Hirosaki University School of Medicine, 5 Zaifu-cho, Hirosaki, Aomori 036-8562, Japan

E-mail: yoshiomi@cc.hirosaki-u.ac.jp

Key words: carboplatin, chemoradiotherapy, dexamethasone, etoposide, extranodal natural killer/T-cell lymphoma, ifosfamide
CRT with 50 Gy/25 fx and 3 cycles of DeVIC comprising non-MDR agents and etoposide for nasal NKTCL.

\section{Introduction}

The nasal type of extranodal natural killer (NK)/T-cell lymphoma (NKTCL) is a rare aggressive lymphoma with a poor prognosis and is more commonly encountered in East Asia (1). Nasal NKTCL refers to tumors arising in the nose, paranasal sinuses and nasopharynx $(2,3)$. It accounts for $3 \%$ of malignant lymphomas in Japan and it is an Epstein-Barr virus-associated lymphoma (4-6). The lymphoma cells express P-glycoprotein, which results in multidrug resistance (MDR) of the tumors (7-10). The disease primarily affects middle-aged males. Nasal bleeding, nasal congestion, rhinitis and facial swelling are the predominant symptoms of this disease. Treatment approaches include radiotherapy (RT), chemotherapy, or a combination of the two. Complete remission was observed in $\sim 72-78 \%$ of patients with RT alone; however, 50-60\% of these cases eventually relapsed (11-15). The reported 5-year overall survival rate for patients with localized nasal NKTCL treated with cyclophosphamide, hydroxydaunorubicin, oncovin and prednisone (CHOP) was $<50 \%$ (16-18). Treatment results of stage I-II disease have recently improved; however, a standard treatment has yet to be established. Dexamethasone, etoposide, ifosfamide and carboplatin (DeVIC) chemotherapy, which was designed as a salvage chemotherapeutic regimen for aggressive lymphomas, comprises non-MDR agents and etoposide and is considered to be effective against nasal NKTCL $(19,20)$. Currently, we are experimenting with chemoradiotherapy (CRT) with DeVIC as the concurrent chemotherapeutic agents for nasal NKTCL. The present study aimed to evaluate the initial outcomes with this treatment and to assess its effectiveness and feasibility.

\section{Materials and methods}

General. Six patients with nasal NKTCL underwent CRT at Hirosaki University Hospital (Hirosaki, Japan) and Aomori Prefectural Hospital (Aomori, Japan) between April, 2004 and February, 2010. The median follow-up time was 56 months (range, 11-80 months). All 6 patients were staged according 
to the Ann Arbor staging criteria. Complete patient evaluation included physical examination, blood counts, screening blood chemistry tests (such as hepatic and renal function and lactate dehydrogenase tests), chest radiographs, whole-body computed tomography (CT) and/or positron emission tomography (PET)-CT scans and bone marrow biopsies.

Patient characteristics. The study included 4 males and 2 females with a median age of 68 years (age range, 29-82 years). In all 6 patients, the primary tumor originated in the nasal cavity. In 5 patients, the tumor involved the nasal cavity unilaterally and in 1 patient it involved the nasal cavities bilaterally and the paranasal sinuses. Five patients had stage IE disease and 1 patient had stage IIE disease. The most frequent presenting symptom was nasal congestion, which was observed in 5 patients. B symptoms were not observed in any of the patients. Elevated lactate dehydrogenase levels were observed in 3 patients. The ratio for performance status (PS) was 0:1 = 3:3 and for International Prognostic Index score was low:low-intermediate $=3: 3$ (Table I).

Chemotherapy. The patients underwent 3-6 cycles of full-dose DeVIC regimen. The drug doses and administration schedule were as follows: dexamethasone (40 mg/day on days 1-3), etoposide (100 $\mathrm{mg} / \mathrm{m}^{2}$ on days $\left.1-3\right)$, ifosfamide $\left(1.5 \mathrm{mg} / \mathrm{m}^{2}\right.$ on days 1-3) and carboplatin (300 $\mathrm{mg} / \mathrm{m}^{2}$ on day 1$)$. The chemotherapy was administered concurrently with RT and was repeated every 3 weeks (Table II).

Radiotherapy. The patients received RT from a linear accelerator using 4-MV X-rays. The clinical target volume included the gross tumor volume and the entire nasal cavity and ipsilateral paranasal sinus. The patients underwent RT with a conventional fractionation schedule at a median dose of 50 Gy (range, 46-50 Gy) (Table II). A three-dimensional dose distribution for a patient with a large tumor is shown in Fig. 1.

\section{Results}

The present study included 4 males and 2 females with a median age of 68 years (age range, 29-82 years) who were treated with CTR using DeVIC in order to assess its effectiveness. Following treatment, the patients were followed up for a median of 56 months (range, 11-80 months).

Complete remission was achieved in 5 patients $(83 \%)$ after 1 month of treatment. The 5-year overall survival and disease-free survival rates were $100 \%$ (Figs. 2 and 3). Although grade 1-2 radiation-induced mucositis of the nasal and oral cavities was observed in 3 patients, no severe adverse effects (grade 3 ) have yet been reported in any of the patients. Two representative cases are shown in Figs. 4 and 5. CT scans prior to CRT reveal a naso-paranasal and a right nasal space-occupying mass of soft tissue. However, 1 month following the completion of CRT, the mass of soft tissue completely disappeared.

\section{Discussion}

Following treatment with DeVIC and CRT, the results showed the complete remission rate to be $83 \%$, which was higher compared to that reported by previous studies on chemotherapy followed
Table I. Patient characteristics.

\begin{tabular}{|c|c|}
\hline Variables & Value \\
\hline \multicolumn{2}{|l|}{ Age (years) } \\
\hline Range & $29-82$ \\
\hline Median & 68 \\
\hline \multicolumn{2}{|l|}{ Gender } \\
\hline Male & 4 \\
\hline Female & 2 \\
\hline \multicolumn{2}{|l|}{ Primary site } \\
\hline Unilateral nasal cavity & 5 \\
\hline Bilateral nasal cavities & 1 \\
\hline \multicolumn{2}{|l|}{ Stage } \\
\hline IE & 5 \\
\hline IIE & 1 \\
\hline \multicolumn{2}{|l|}{ B symptoms } \\
\hline Yes & 0 \\
\hline No & 6 \\
\hline \multicolumn{2}{|c|}{ Lactate dehydrogenase elevation } \\
\hline Yes & 3 \\
\hline No & 3 \\
\hline \multicolumn{2}{|l|}{ PS } \\
\hline 0 & 3 \\
\hline 1 & 3 \\
\hline \multicolumn{2}{|l|}{ IPI score } \\
\hline Low & 3 \\
\hline Low-intermediate & 3 \\
\hline
\end{tabular}

PS, performance status; IPI, International Prognostic Index.

Table II. Treatment characteristics.

\begin{tabular}{lc}
\hline Variables & $\begin{array}{c}\text { No. of } \\
\text { patients }\end{array}$ \\
\hline Radiation dose (Gy) & \\
50 & 4 \\
46 & 2 \\
Non-opposing pair & 2 \\
Multiple field & 4 \\
Chemotherapy (cycles) & \\
3 & 3 \\
4 & 2 \\
6 & 1
\end{tabular}

by RT. Sakata et al reported that radiation doses $>52$ Gy may be required to obtain local control in patients with localized nasal NKTCL (21). Koom et al reported that radiation doses <45 Gy were significantly associated with local relapse (12). These studies have been cited by several textbooks. Our median radiation dose 


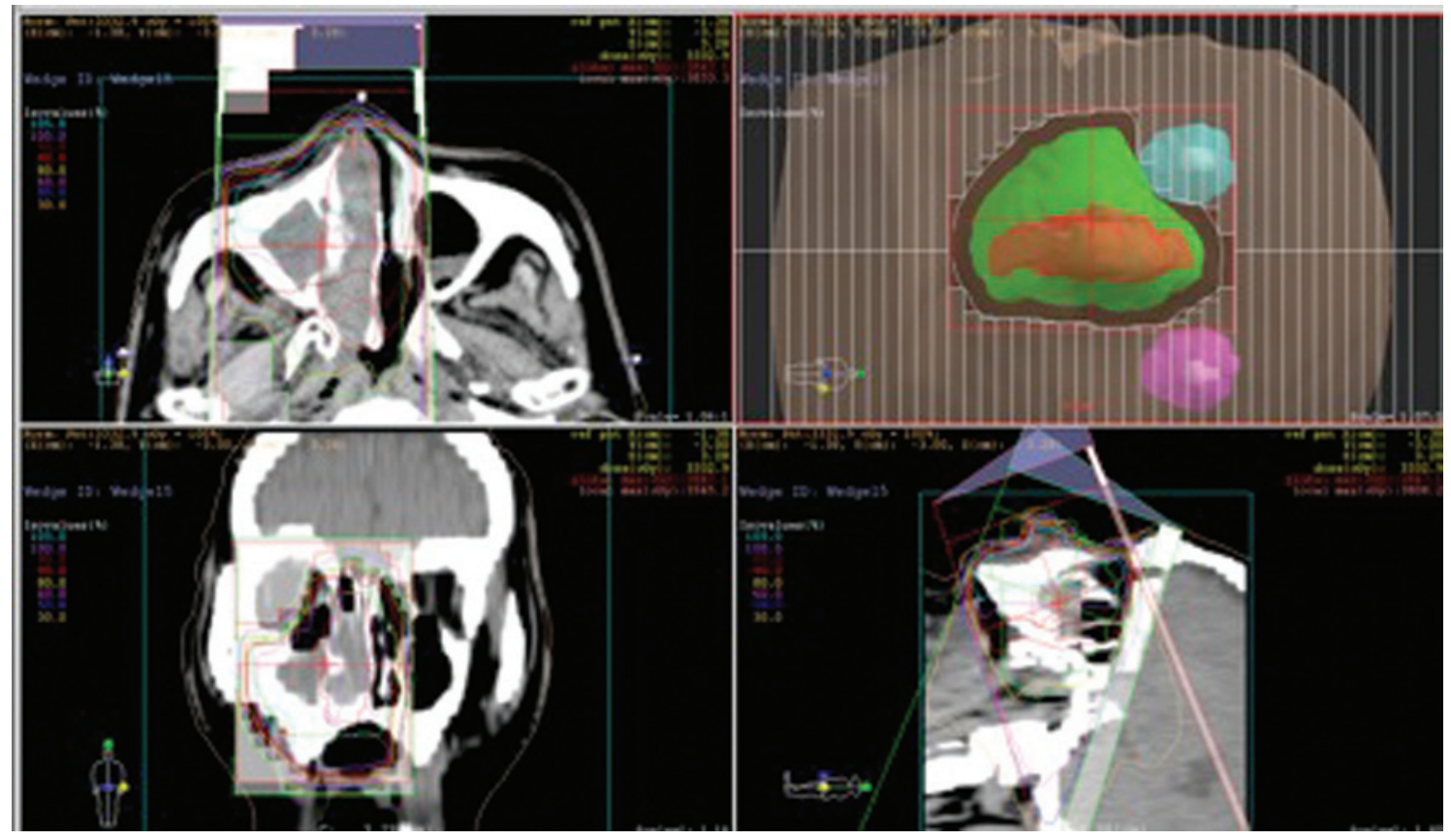

Figure 1. Three-dimensional dose distributions for a patient who received a total dose of 50 Gy with two fixed non-coplanar conformal beams. Volume: red, GTV; green, CTV.
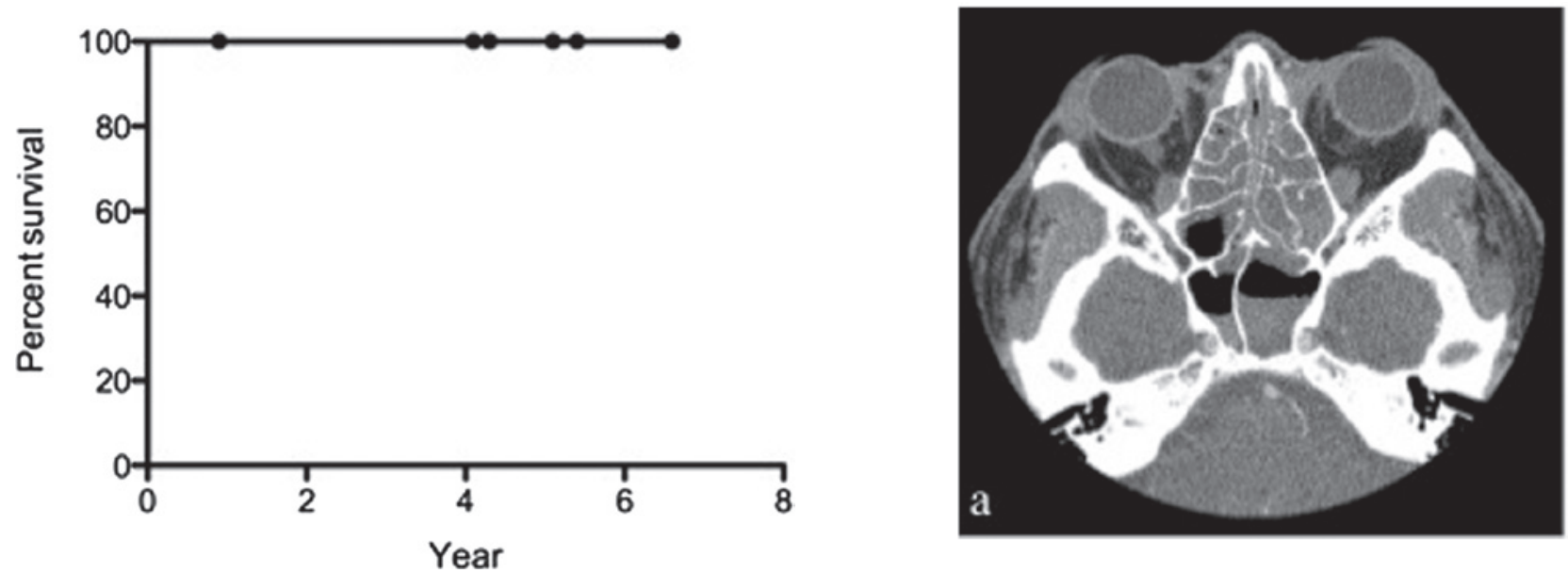

Figure 2. Overall survival.
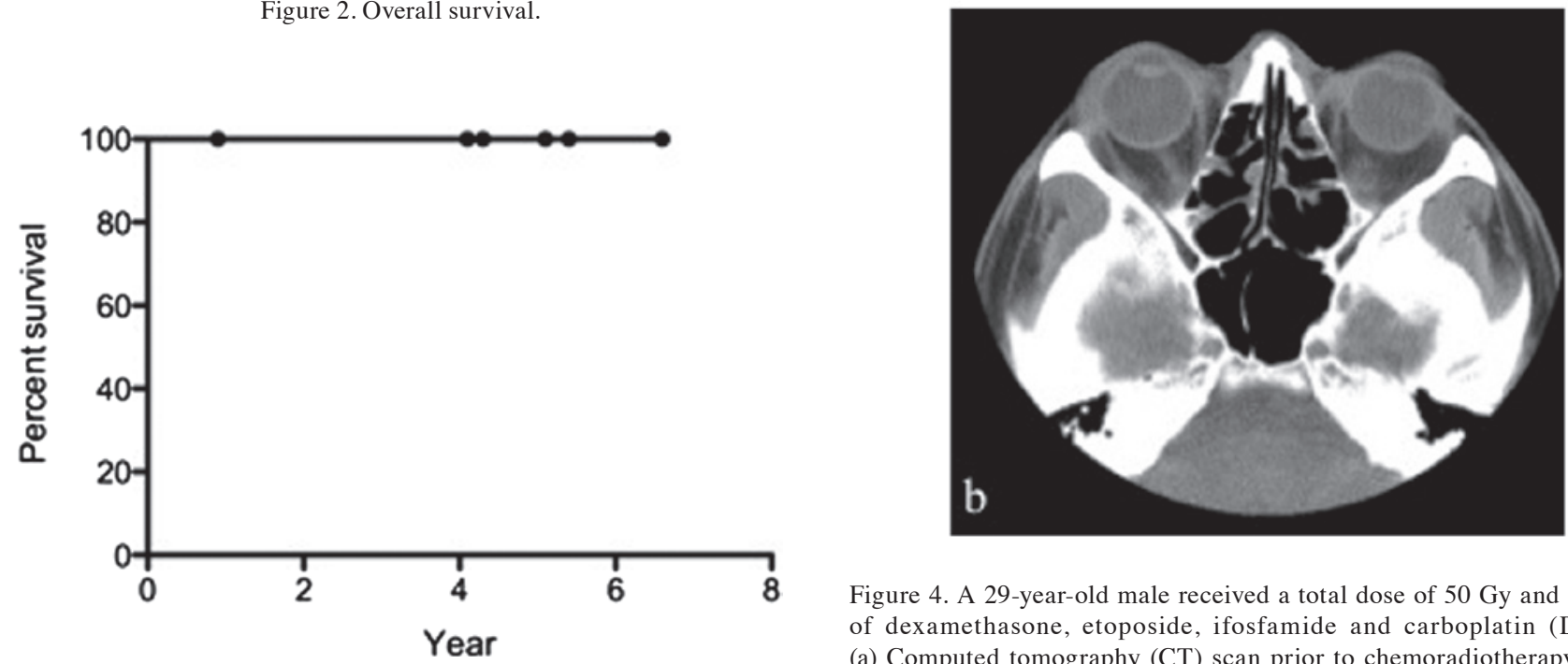

Figure 4. A 29-year-old male received a total dose of 50 Gy and 6 cycles of dexamethasone, etoposide, ifosfamide and carboplatin (DeVIC). (a) Computed tomography (CT) scan prior to chemoradiotherapy (CRT) shows a naso-paranasal space-occupying soft tissue mass. (b) The soft tissue Figure 3. Disease-free survival. mass completely disappeared 1 month after the completion of CRT. 

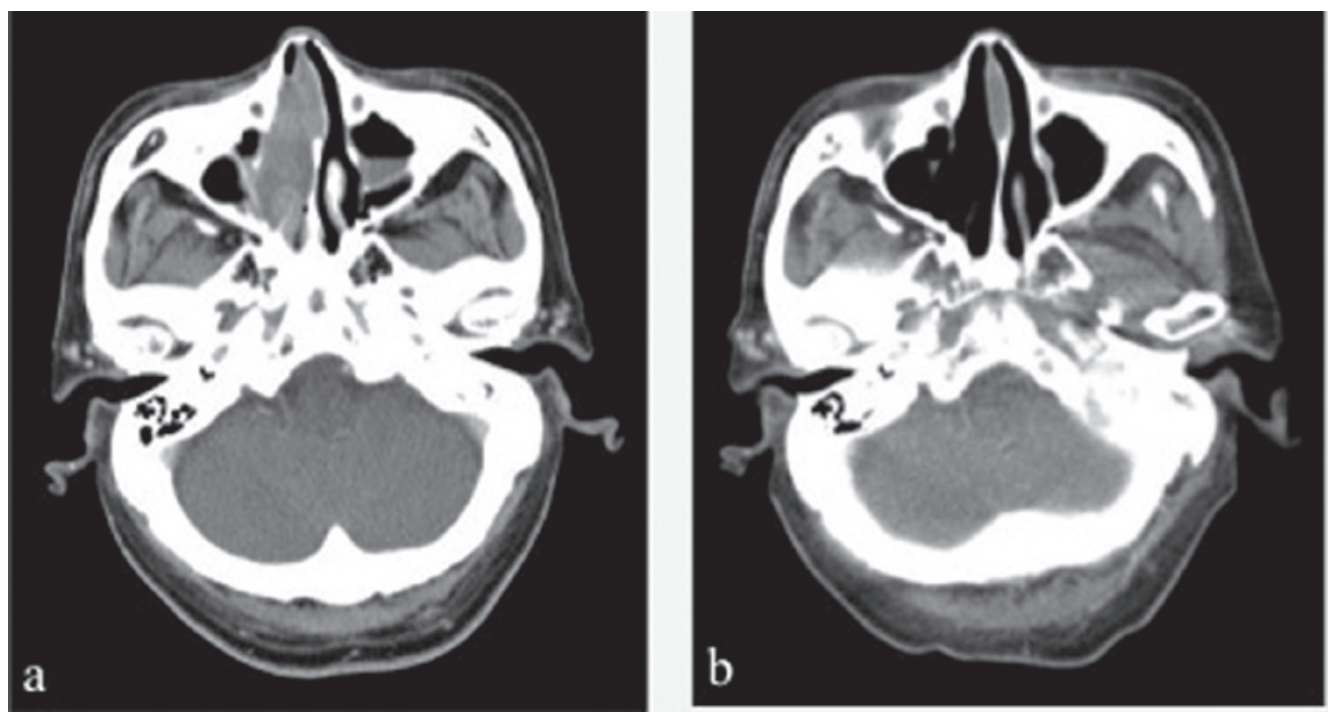

Figure 5. A 72-year-old male received a total dose of $50 \mathrm{~Gy}$ and 4 cycles of dexamethasone, etoposide, ifosfamide and carboplatin (DeVIC). (a) Computed tomography (CT) scan prior to chemoradiotherapy (CRT) shows a right nasal space-occupying soft tissue mass. (b) The mass completely disappeared 1 month after the completion of CRT.

was 50 Gy (range, 46-50 Gy). However, Kim et al reported satisfactory local control with lower doses of $\sim 40 \mathrm{~Gy}(22)$.

DeVIC was added to the regimen for management of systemic relapse and consolidation. This regimen has been used in combination with RT since the late 1990s in Japan (20). All the patients received 3-6 cycles of a full-dose DeVIC regimen and 46-50 Gy of RT.

Severe mucositis and moderate dermatitis are the side effects of CRT in the nasal cavity; therefore, nutritional management and pain control are critical. In addition, the radiation dose to the optic nerve, optic chiasm and eyeball must be considered in order to avoid visual disorders. All patients completed the treatment without severe adverse events. To date, no visual disorders have been reported.

In previous retrospective studies, the local control and systemic relapse rates of nasal NKTCL were $18.9-47 \%$ and $15.1-81 \%$, respectively $(12,13,23,24)$. In those studies, the patients were either treated with RT alone or a combination of RT and CHOP-based chemotherapy. The 5-year survival rate for localized nasal NKTCL is $47-57 \%(17,18)$.

Thus far no local recurrence or systemic relapse has been observed in any of the patients. All 6 patients were still alive at the time of writing this manuscript. Our initial results from the present experimental CRT/DeVIC regimen for this aggressive type of lymphoma were encouraging. Additional investigation is required on concurrent CRT with $50 \mathrm{~Gy} / 25 \mathrm{fx}$ and 3-6 cycles of DeVIC comprising non-MDR agents and etoposide for nasal NKTCL.

Our study included patients who were under a short period of observation and our results suggest that the use of CRT with DeVIC is effective for the treatment of nasal NKTCL.

\section{References}

1. No authors listed: The World Health Organization classification of malignant lymphomas in Japan: incidence of recently recognized entities. Lymphoma Study Group of Japanese Pathologists. Pathol Int 50: 696-702, 2000.
2. Chan JK, Quintanilla-Martinez L, Ferry JA, et al: Extranodal NK/T-cell lymphoma, nasal type. In: WHO Classification of Tumours of Haematopoietic and Lymphoid Tissues. Swerdlow SH, Campo E, Harris NL, et al (eds). IARC Press, Lyon, pp285-288, 2008.

3. Al-Hakeem DA, Fedele S, Carlos R and Porter S: Extranodal NK/T-cell lymphoma, nasal type. Oral Oncol 43: 4-14, 2007.

4. Suzuki R, Takeuchi K, Ohshima K and Nakamura S: Extranodal NK/T-cell lymphoma: diagnosis and treatment cues. Hematol Oncol 26: 66-72, 2008.

5. Harabuchi Y, Yamanaka N, Kataura A, et al: Epstein-Barr virus in nasal T-cell lymphomas in patients with lethal midline granuloma. Lancet 335: 128-130, 1990.

6. Jaffe ES, Chan JK, Su IJ, et al: Report of the workshop on nasal and related extranodal angiocentric T/Natural killer cell lymphomas. Definitions, differential diagnosis, and epidemiology. Am J Surg Pathol 20: 103-111, 1996.

7. Jung CK, Lee KY, Kim Y, et al: Epstein-Barr virus infection, drug resistance and prognosis in Korean T- and NK-cell lymphomas. Pathol Int 51: 355-363, 2001.

8. Yamaguchi M, Kita K, Miwa $\mathrm{H}$, et al: Frequent expression of P-glycoprotein/MDR1 by nasal T-cell lymphoma cells. Cancer 76: 2351-2356, 1995.

9. Drenou B, Lamy T, Amiot L, et al: CD3-CD56 ${ }^{+}$non-Hodgkin's lymphomas with an aggressive behavior related to multidrug resistance. Blood 89: 2966-2974, 1997.

10. Egashira M, Kawamata N, Sugimoto K, Kaneko T and Oshimi K: P-glycoprotein expression on normal and abnormally expanded natural killer cells and inhibition of P-glycoprotein function by cyclosporine A and its analogue, PSC 833. Blood 93:599-606,1999.

11. Kim GE, Cho JH, Yang WI, et al: Angiocentric lymphoma of the head and neck: patterns of systemic failure after radiation treatment. J Clin Oncol 18: 54-63, 2000.

12. Koom WS, Chung EJ, Yang WI, et al: Angiocentric T-cell and NK/T-cell lymphomas: radiotherapeutic viewpoints. Int J Radiat Oncol Biol Phys 59: 1127-1137, 2004.

13. Cheung MM, Chan JK, Lau WH, Ngan RK and Foo WW: Early stage nasal NK/T-cell lymphoma: clinical outcome, prognostic factors, and the effect of treatment modality. Int J Radiat Oncol Biol Phys 54: 182-190, 2002.

14. Kim WS, Song SY, Ahn YC, et al: CHOP followed by involved field radiation: is it optimal for localized nasal natural killer/T-cell lymphoma? Ann Oncol 12: 349-352, 2001.

15. Liang R, Todd D, Chan TK, et al: Treatment outcome and prognostic factors for primary nasal lymphoma. J Clin Oncol 13: 666-670, 1995.

16. Ribrag V, Ell Hajj M, Janot F, et al: Early locoregional high-dose radiotherapy is associated with long-term disease control in localized primary angiocentric lymphoma of the nose and nasopharynx. Leukemia 15: 1123-1126, 2001. 
17. Shikama N, Ikeda H, Nakamura S, et al: Localized aggressive non-Hodgkin's lymphoma of the nasal cavity: a survey by the Japan Lymphoma Radiation Therapy Group. Int J Radiat Biol Phys 51: 1228-1233, 2001.

18. Isobe K, Uno T, Tamaru J, et al: Extranodal natural killer/T-cell lymphoma, nasal type: the significance of radiotherapeutic parameters. Cancer 106: 609-615, 2006.

19. Yamaguchi M, Tobinai M, Oguchi M, et al: Phase I/II study of concurrent chemoradiotherapy for localized nasal NK/T-cell lymphoma: Final results of JCOG0211. J Clin Oncol 27: 5594-5600, 2009.

20. Yamaguchi M, Ogawa S, Nomoto Y, et al: Treatment outcome of nasal NK-cell lymphoma: a report of 12 consecutively-diagnosed cases and a review of the literature. J Clin Exp Hematopathol 41: 93-99, 2001.
21. Sakata K, Fuwa N, Kodaira T, et al: Analyses of dose-response in radiotherapy for patients with mature T/NK-cell lymphomas according to the WHO classification. Radiother Oncol 79: 179-184, 2006

22. Kim SJ, Kim K, Kim BS, et al: Phase II trial of concurrent radiation and weekly cisplatin followed by VIPD chemotherapy in newly diagnosed, stage IE to IIE, nasal, extranodal NK/T-cell lymphoma: consortium for improving survival of lymphoma study. J Clin Oncol 27: 6027-6032, 2009.

23. Li YX, Yao B, Jin J, et al: Radiotherapy as primary treatment for stage IE and IIE nasal natural killer/T-cell lymphoma. J Clin Oncol 24: 181-189, 2006.

24. Wang B, Lu JJ, Ma X, et al: Combined chemotherapy and external beam radiation for stage IE and IIE natural killer T-cell lymphoma of nasal cavity. Leuk Lymphoma 48: 396-402, 2007. 